\title{
Is Good Good Enough?
}

\author{
David W. Roberts
}

Published online: 12 February 2009

(C) Humana Press Inc. 2009

Ventriculostomy for cerebrospinal fluid diversion, either externalized as a temporary drain or internalized as a permanent shunt, is a frequently performed, clinically invaluable procedure. In this issue, two studies concerning the accuracy and morbidity of this procedure are reported: one a large, single institution's 5-year experience with external ventricular drains and ventricular shunts placed by their neurosurgical service, and the other, a smaller, 3-year experience with external ventricular drain and intracranial pressure monitor placement by a single, specially trained neurointensivist. The former documents a well-established service's track record, contributing to the literature's collective benchmarking experience; the latter also documents an institution's track record, but in this instance, as part of an effort to validate the practice of non-neurosurgical neurointensivists engaging in this activity.

In the paper by Saladino et al. [1] from the Mayo Clinic, 212 ventriculostomy procedures performed by neurosurgeons in a total of 138 patients are described. Hemorrhage related to the procedure was seen in $15(7.1 \%)$ patients, in 4 of whom this was symptomatic. Twenty-six (12.3\%) ventricular catheters were malplaced (using low-threshold criteria), with 21 intraparenchymal and 5 extraventricular. Infections related to the procedure were seen in $7(3.3 \%)$. Neurological sequelae were rare, and the authors conclude by observing the relatively low risk associated with the procedure [1].

Ehtisham et al. [2] from the University of Kansas report their experience with 29 external ventricular drain and 7 intracranial pressure monitor placements in a neurological

D. W. Roberts ( $)$

Dartmouth-Hitchcock Medical Center, Lebanon, NH 03756,

USA

e-mail: david.w.roberts@dartmouth.edu critical care unit. Six (20.7\%) of the external ventricular drains were associated with hemorrhage, none of which was symptomatic. There were no infections. No procedurerelated complications were observed in the seven intracranial pressure monitor placements. The authors conclude that this experience suggests that external ventricular drain and intracranial pressure monitor placement by neurointensivists may be safe and effective [2].

There is a fairly wide range of successful catheter placement and associated morbidities in the literature. Hemorrhage rates between 1 and 33\% and infection rates as high as $12 \%$ have been reported, and both of these current reports not surprisingly fall within the expectations set by this collective experience. Comparative analyses of this literature, including both new contributions, are particularly difficult on account of retrospective methodologies, nonuniform definitions of accuracy and of various morbidities, and small numbers. Reporting bias that may favor better outcomes only further confounds the issue.

It is likely that the bulk of discussion generated by these two most recent studies will focus on matters of professional turf raised by the prospect of neurointensivists increasingly participating in a heretofore neurosurgical procedure. Thirty-six procedures by one individual are hardly compelling, but questions of validation almost certainly will ultimately be answered by data. There is no doubt that training and special focus can generate efficient expertise: one need look no further than the blood-draw and i.v. teams in every hospital. There are aspects to intracranial procedures, on the other hand, that make the discussion more complex. Are any distinctions drawn along the continuum of technical difficulty (e.g., midline shift, small ventricles, associated parenchymal pathology)? What judgment regarding indication, independent of technical proficiency, is expected? How is coordination of such 
intervention with an overall treatment plan (e.g., timing or location of a permanent shunt) achieved? What responsibilities and capabilities with respect to handling of complications related to the procedure should be expected? Is the promptness afforded by a $24 / 7$ presence in a critical care unit really an issue? How is special training in the procedure to be accomplished? Are there sufficient numbers of procedures to train and maintain proficiency for members of all subspecialties with interest in the procedure? These are just some of the questions that must be addressed. Ultimately of course, the matter should not be specialty-driven but patient-centric. What is in the best interest of the patient, not our respective guilds, should and must have primacy.

Beyond the controversies of professional territory, however, these papers raise a seldom expressed but perhaps more profound concern. For decades, neurosurgeons have routinely placed ventricular catheters for external drainage or permanent shunts, using their hard-earned and timetested, freehand technical abilities, the results of which are alluded to above in a wide if retrospective literature. We have accepted today's results, benchmarking against that broad-ranging experience. Perhaps the more important question we should be asking is whether we should be satisfied with an $88 \%$ success rate in placing a ventricular catheter where intended, let alone the generally undocumented number of passes required to achieve that rate (and I suspect this is high compared with unpublished performance). Acknowledgedly, misplaced catheters appear to be remarkably well tolerated-but not always. Can the success rate be higher? Hemorrhage rates, although distinctly different from those of accuracy, do likely track with the number of trajectories and difficulty of placement. Should we be satisfied with a $7 \%$ or a $20 \%$ incidence of hemorrhage? Can it be lower?
The answer may not yet be available for hemorrhage, but it is for accuracy of catheter location. Simply monitoring and tracking will improve most performance, and attention to catheter placement will likely have a similar effect. For more difficult ventricular catheter placement, utilization of available technology-in this instance, image-guidance-has been demonstrated to be effective. McGirt et al. [3] reported use of image guidance to successfully place ventricular shunts in 36 patients with pseudotumor cerebri (a patient population characterized by very small ventricular size). Lollis and Roberts [4] more recently reported successful placement of ventricular catheters in 16 patients, many of whom had small ventricles, using a robotic, image-guidance technique. Catheters were successfully placed in all patients with a single pass, with a mean distance of catheter tip from target of $1.5 \mathrm{~mm}$; there were no hemorrhages. Although not prospective, double-blind studies, such reports suggest a potential paradigm shift or at least a change in expectations. A meaningful debate might revolve around what allocation of such technological resources should be made.

\section{References}

1. Saladino A, White JB, Wijdicks FM, et al. Malplacement of ventricular catheters by neurosurgeons: a single institution experience. Neurocrit Care. 2008;in press.

2. Ehtisham A, Taylor S, Bayless L, et al. Placement if external ventricular drains and intracranial pressure monitors by neurointensivists. Neurocrit Care. 2008;in press.

3. McGirt MJ, Woodworth G, Thomas G, et al. Cerebrospinal fluid shunt placement for pseudotumor cerebri-associated intractable headache: predictors of treatment response and an analysis of longterm outcomes. J Neurosurg. 2004;101:627-32.

4. Lollis SS, Roberts DW. Robotic catheter ventriculostomy: feasibility, efficacy, and implications. J Neurosurg. 2008;108:269-74. 